\title{
Diagnosis of pulmonary embolism in hospitalised patients: retrospective survey of an institutional standard
}

\author{
S. Jouveshomme*, I. Bohn* and A. Cazaban\#
}

ABSTRACT: The aim of the present study was to assess the safety and implementation of a diagnostic strategy in hospitalised patients with suspected acute pulmonary embolism (PE).

A diagnostic strategy was established and implemented in a general hospital. A retrospective cohort study, including 400 consecutive in-patients, was performed in order to assess the appropriateness of the diagnostic management and the incidence of symptomatic venous thromboembolic events (VTE) during follow-up.

PE was confirmed in $116(29 \%)$ patients. The incremental value of adding compression ultrasonography (CUS) to multidetector-row computed tomography (MDCT) for the diagnosis of PE was $8.6 \%$. PE was appropriately excluded in 169 (42\%) patients due to a normal lung scan $(n=34)$, a negative MDCT providing an alternative diagnosis $(n=94)$, and a negative MDCT and CUS $(n=41)$. During follow-up, VTE occurred in $3.5 \%$ patients. The almost unique cause of inappropriate management was the absence of further work-up after a MDCT-negative result for PE providing no alternative diagnosis $(n=115)$. Inappropriate management was associated with a nonsignificant increased risk of VTE (7.2\%).

A frontline diagnostic work-up based on pulmonary multidetector-row computed tomography associated with a compression ultrasonography of the leg veins is effective and more sensitive than pulmonary multidetector-row computed tomography alone in ruling out pulmonary embolism.

KEYWORDS: Compression ultrasonography, computed tomography, deep vein thrombosis, implementation study, pulmonary embolism

$\mathbf{T}$ he diagnosis of pulmonary embolism (PE) is challenging because the signs and symptoms are not specific [1] and because no single noninvasive test is suitable in all cases. During the past decade several diagnostic strategies, based on the combination of clinical probability and one or several noninvasive diagnostic tests, have been validated in outpatients [2]. It has recently been shown that nonadherence to validated diagnostic guidelines exposes patients to increased risk for a subsequent venous thromboembolic event (VTE) [3].

As many as $40 \%$ of PEs occur in in-patients [4] but little is known about the optimal diagnostic strategy in this population. Moreover, current diagnostic guidelines may not be suitable for inpatients, since clinical probability scores have not been validated in this population, and some tests, such as D-dimer testing, have been found to be inappropriate in this setting [5-7].
Helical computed tomography (CT) has rapidly gained acceptance as the frontline imaging mode for the diagnosis of PE for the following reasons: 1) it is a relatively noninvasive procedure that can be used to diagnose PE by directly imaging the intravascular clot; and 2) it can provide an alternative diagnosis to explain a patient's signs and symptoms. However, sensitivity of singledetector $\mathrm{CT}$ is too low to detect isolated peripheral $\mathrm{PE}$, and patients with a negative single-detector CT providing no alternative diagnosis need additional imaging tests to rule out PE definitively [8]. The development of multidetector-row CT (MDCT) during the past few years allows analysis of pulmonary arteries down to the sixth subdivision and has improved the accuracy of CT in diagnosing PE [9]. Recent studies have shown that anticoagulation can be safely withheld in outpatients with suspected PE but a negative MDCT $[10,11]$. However, little is known about the use of MDCT in hospitalised patients.

\section{AFFILIATIONS}

*Pneumology and Thoracic Oncology Service, and \#Radiology Service, CHI Poissy-St Germain, St Germain-en-Laye, France.

CORRESPONDENCE

S. Jouveshomme

Service de Pneumologie et

d'Oncologie Thoracique

CHI Poissy-St Germain

20, rue d'Armagis

78100 St Germain-en-Laye

France

Fax: 33139274162

E-mail: stephanejouveshomme@

chi-psg.com

Received:

February 222007

Accepted after revision:

August 032007

STATEMENT OF INTEREST

None declared.

European Respiratory Journal Print ISSN 0903-1936 Online ISSN 1399-3003 
Although this is still a matter of some debate, PE and deep venous thrombosis (DVT) are widely thought to be two clinical manifestations of the same disease. Indeed, DVT is found in $\leqslant 80 \%$ of patients with acute $\mathrm{PE}$ [12] and positive compression ultrasonography (CUS) of the lower limb veins has been shown to be highly predictive of PE on MDCT [13]. Consequently, testing for DVT has become an integral part of the diagnosis work-up of PE [14].

The present authors have established, by consensus meetings involving cardiologists, pulmonologists and radiologists, a best practice clinical guideline for the diagnosis of PE in in-patients, mainly based on MDCT and lower limb CUS. Following implementation of this guideline in the present authors' hospital (CHI, Poissy-St Germain, St Germain-en-Laye, France), a retrospective cohort has been formed in order to determine the following: 1) the adherence to the proposed diagnostic strategy; 2) the 3-month outcome of patients considered negative for PE; 3 ) whether outcome was correlated with adherence to the recommended diagnostic strategy; and 4) the incremental value of CUS performed after a negative MDCT.

\section{METHODS}

\section{Institutional guidelines}

D-dimer testing was not included in the present authors' diagnostic algorithm due to its low yield in hospitalised patients. Therefore, the firstline diagnostic strategy was independent of the clinical probability of PE. MDCT of the pulmonary arteries was the frontline diagnostic test in the composite reference standard. A normal or inconclusive MDCT that did not show an alternative diagnosis able to explain a patient's signs or symptoms was followed by CUS. Confirmation of a negative result of both MDCT and CUS by a perfusion lung scan or a pulmonary digital-subtraction angiography was left to the discretion of the attending physician. The finding of DVT on CUS established a classifying diagnosis of PE and anticoagulation was started. In patients with suspected PE but impaired renal function, CUS was considered as the firstline diagnostic test. A negative result was followed by MDCT after appropriate hydration. Finally, a normal perfusion lung scan was accepted to rule out PE.

\section{Diagnostic studies}

MDCT of the pulmonary arteries was performed with a 16-detector-CT (Sensation 16; Siemens, Erlingen, Germany). A $100 \mathrm{~mL}$ aliquot of low osmolar non-ionic contrast material was injected through an arm vein at $3.5 \mathrm{~mL} \cdot \mathrm{s}^{-1}$. Injection to scan delay was determined by bolus tracking. Images were obtained from the diaphragm to the top of the thorax, with a section width of $16 \times 0.75 \mathrm{~mm}$. All examinations were displayed and read from high-quality workstations by experienced CT radiologists. Bilateral venous CUS of the leg veins was performed from the common femoral vein down to the trifurcation of the calf veins included. A lack of compressibility was considered to indicate DVT. Perfusion lung scans were performed with at least six views (anterior, posterior, right and left oblique posterior, right and left oblique lateral). Lung scans were interpreted as normal according to the Prospective Investigation of Pulmonary Embolism Diagnosis (PIOPED) criteria [15].

\section{Study population}

According to French regulations, a participant's written informed consent is not required for observational studies. All patients hospitalised for $\geqslant 24 \mathrm{~h}$ in the present authors' 625-bed general hospital, and referred for diagnostic imaging (perfusion lung scan, CUS of the legs, MDCT of the pulmonary arteries) for clinically suspected PE between December 2002 and February 2005, were retrospectively identified through the computerised radiology information system (Xplore ${ }^{\circledR}$ 6.2.3; EDL, Berre l'Etang, France). Exhaustivity of the patient selection, assessed by comparing the PE study population with the International Classification of Diseases database of the present authors' hospital, was 91\%. Medical records were reviewed for each patient. The clinical information recorded included symptoms of PE, risk factors for VTE, the strategy used to diagnose or exclude PE, the final report of the interpreting radiologist, the alternative diagnosis provided by an MDCT considered negative for PE, and indications of anticoagulation therapy for a reason other than VTE.

\section{Three-month follow-up}

To confirm the safety of the exclusion of PE, attempts were made between June and September 2005 to locate all patients with a negative study result in order to determine the 3-month outcomes (either death or VTE within the 3 months following the first diagnostic test). Hospital records were reviewed and patients, their families or caregivers were contacted. VTE was considered to be confirmed if: the diagnosis was objectively documented by a spiral CT; CUS showed DVT; results of pulmonary angiography were positive; or an autopsy confirmed the diagnosis. All deaths were reviewed by at least two study reviewers to determine whether PE may have occurred based on all available evidence. Unexpected and otherwise unexplained deaths were attributed to PE.

\section{Statistical analysis}

A Chi-squared test was used to assess the association between the appropriateness of the diagnostic testing and categorical variables, with a two-sided significance level of $\mathrm{p}<0.05$.

\section{RESULTS}

\section{Study population}

A total of 400 in-patients referred for diagnostic imaging for clinically suspected PE during the study period were included. Their mean \pm SD age was $66 \pm 20$ yrs and $44 \%$ were male. No patient was excluded due to incomplete medical records. Clinical presentation and risk factors for VTE are shown in table 1. MDCT, CUS and perfusion lung scan were performed in $363(91 \%), 82(21 \%)$ and $46(12 \%)$ patients, respectively (fig. 1). No pulmonary digital-subtraction angiography was performed. Among the 37 patients who did not undergo MDCT, five had severe renal insufficiency (creatinine clearance $\left.<30 \mathrm{~mL} \cdot \mathrm{min}^{-1}\right)$. PE was diagnosed in $116(29 \%)$ patients. The criteria used for diagnosis or exclusion of PE are shown in table 2.

\section{PE confirmed}

PE was confirmed by a positive MDCT in 96 patients (83\%). A total of 10 patients $(9 \%)$ had a negative MDCT but had DVT (eight proximal and two calf vein thrombosis) at CUS. In one additional patient (1\%), MDCT was considered nondiagnostic 
TABLE 1 Characteristics of the 400 patients in the present study

\begin{tabular}{|c|c|c|c|c|}
\hline & \multirow[t]{2}{*}{ Total } & \multirow[t]{2}{*}{ PE diagnosed } & \multicolumn{2}{|c|}{ PE excluded } \\
\hline & & & Yes & No \\
\hline Subjects $n$ & 400 & 116 & 169 & 115 \\
\hline \multicolumn{5}{|l|}{ Characteristic } \\
\hline \multicolumn{5}{|l|}{ Risk factors for VTE } \\
\hline Family history of VTE & $5(1)$ & $2(2)$ & $3(2)$ & 0 \\
\hline Previous VTE & $62(15)$ & $23(20)$ & $28(17)$ & $11(10)$ \\
\hline Known heart failure & $54(13)$ & $7(6)$ & $30(18)$ & $17(15)$ \\
\hline Stroke & $4(1)$ & $2(2)$ & $2(1)$ & 0 \\
\hline \multicolumn{5}{|l|}{ Symptoms of PE } \\
\hline Dyspnoea & $296(74)$ & $87(76)$ & & \\
\hline Chest pain & 167 (42) & $52(45)$ & & \\
\hline Haemoptysis & $6(2)$ & $3(3)$ & & \\
\hline Recent cough & $1(0)$ & $0(0)$ & & \\
\hline Syncope or malaise & $16(4)$ & $2(2)$ & & \\
\hline Unexplained fever & $13(3)$ & $2(2)$ & & \\
\hline \multicolumn{5}{|l|}{ Patient recruitment area } \\
\hline Pneumology ward & $148(37)$ & $37(32)$ & & \\
\hline Cardiology ward & $126(36)$ & $47(40)$ & & \\
\hline Medicine ward & $83(18)$ & $18(16)$ & & \\
\hline
\end{tabular}

Data are presented as $n(\%)$, unless otherwise indicated. PE: pulmonary embolism; VTE: venous thromboembolic events; COPD: chronic obstructive pulmonary disease.

but CUS showed DVT. In eight (7\%) patients, PE was diagnosed on the basis of a clinical suspicion of $\mathrm{PE}$ and a positive CUS, without further confirmation by MDCT. In one (1\%) patient, the diagnosis of PE was based only on an abnormal perfusion lung scan. The diagnostic criteria for PE were in accordance with the reference standard in 115 (99\%) patients. The incremental value of adding CUS to MDCT for the diagnosis of $\mathrm{PE}$ was $\geqslant 8.6 \%$ (95\% confidence interval (CI) 3.5-13.7).

\section{PE excluded}

Among the 284 patients considered negative for PE, MDCT was performed in 262 (92\%). In 94 (33\%) patients, MDCT provided an alternative diagnosis able to explain a patient's signs or symptoms. Among the 169 (60\%) patients with an MDCT negative for PE providing no alternative diagnosis, CUS showed no DVT in $41(14 \%)$ and a perfusion lung scan was considered normal in three (2\%) patients, leaving 115 $(40 \%)$ patients with no additional diagnostic test being performed. In $31(11 \%)$ additional patients, PE was ruled out by a normal perfusion lung scan, including three patients with an MDCT considered nondiagnostic. The criteria used to rule out PE were in accordance with the reference standard in $60 \%$ of the patients.

\section{Clinical outcome in patients in whom PE was initially ruled out}

Among the patients considered negative for PE, $34(12 \%)$ received anticoagulation therapy for a reason other than VTE and $10(3.5 \%)$ were lost to follow-up, leaving 240 patients for the assessment of the 3-month VTE risk. A total of $156(65 \%)$ patients were followed in the present authors' institution, whereas follow-up of the remaining patients was obtained by telephone. During the 3-month follow-up period, $25(10 \%)$ patients died. No autopsy was performed but three deaths were adjudicated as possibly being due to PE. The other causes of death were as follows: cancer $(n=11)$, septic shock $(n=5)$, febrile neutropenia $(n=1)$, alveolar haemorrhage $(n=1)$, peritonitis $(n=1)$, acute respiratory distress syndrome $(n=2)$ and Stevens-Johnson syndrome $(n=1)$. PE occurred in six patients and isolated DVT occurred in three patients. Therefore, the 3-month risk of VTE among the leaving 218 patients who did not receive anticoagulation therapy during follow-up was $5.5 \%$ (95\% CI 2.5-8.5). Nonadherence with the reference standard to 


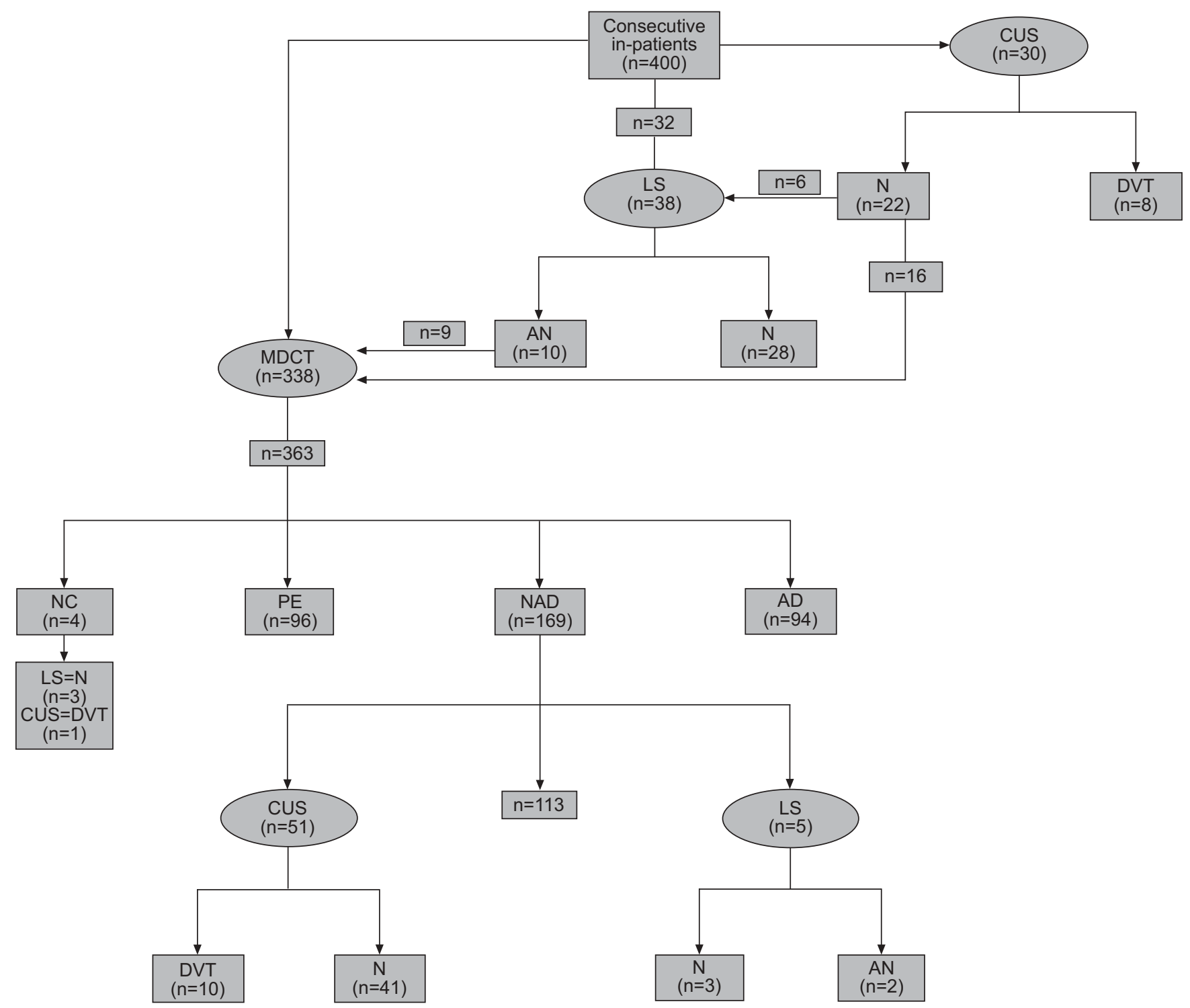

FIGURE 1. Flow chart summarising the diagnostic process used in the present study. PE: pulmonary embolism; MDCT: multidetector-row computed tomography; LS: lung scan; CUS: lower limb compression ultrasonography; DVT: deep venous thrombosis; NC: not contributive; AD: alternative diagnosis; NAD: no alternative diagnosis; $\mathrm{N}$ : normal; AN: abnormal.

exclude PE was associated with a 7.9\% (95\% CI 2.3-13.5) risk of VTE as compared with a $3.8 \%(95 \%$ CI $0.5-7.1)$ rate when the initial diagnostic work-up was performed according to the reference standard $(\mathrm{p}<0.25$; table 3$)$.

\section{DISCUSSION}

The assessment for PE was incomplete in $29 \%$ of the patients in the present study, which is slightly lower than the $43 \%$ reported in a recent cohort study involving 116 emergency departments in France [3]. The almost unique cause of nonadherence to the reference standard in the present study was the lack of CUS of the lower extremities in patients with a MDCT showing neither PE nor an alternative diagnosis. However, when performed, CUS of the lower extremities found DVT in $5.9 \%$ of patients with a negative MDCT, providing an incremental value in the diagnosis of PE of $\geqslant 8.6 \%$. Similar results have been observed in other studies involving in- and outpatients [16, 17]. This lack of adherence to the reference standard was not correlated to the recruitment area of the patients and may be explained by the intuitive confidence of the attending physicians in a negative CT when there is not a high suspicion of PE.

Conversely, data from the present study show that the clinical reference standard is safe to rule out PE in hospitalised patients, with a 3-month risk of VTE of 3.8\% that compares favourably with the few studies that have reported on the outcome of in-patients in this setting [18-20]. When compared with the population of emergency patients with suspected PE included in previous studies, the present study population is older and more likely to have cancer [10, 11, 17]. This may explain why the 3-month risk of VTE presently observed is 
TABLE 2 Basis for the diagnosis or exclusion of pulmonary embolism (PE)

\begin{tabular}{|c|c|c|c|}
\hline Diagnostic criterion & Total & $\begin{array}{l}\text { Accordance with } \\
\text { reference standard }\end{array}$ & Diagnostic failure \\
\hline \multicolumn{4}{|l|}{ PE confirmed } \\
\hline Subjects $n$ & 116 & & \\
\hline MDCT positive & $96(82)$ & Yes & \\
\hline MDCT negative, DVT on CUS & $10(9)$ & Yes & \\
\hline Abnormal perfusion lung scan & $1(1)$ & No & \\
\hline \multicolumn{4}{|l|}{ PE excluded } \\
\hline Subjects $n$ & 284 & & 12 \\
\hline No PE on MDCT but alternative diagnosis & $94(33)$ & Yes & 1 death $^{\#}, 1$ PE \\
\hline No PE or alternative diagnosis on $\mathrm{MDCT}$, CUS negative & $41(14)$ & Yes & 1 death\#, 1 PE, 1 DVT \\
\hline No PE or alternative diagnosis on MDCT, normal perfusion lung scan & $3(1)$ & Yes & \\
\hline
\end{tabular}

Data are presented as $n$ (\%), unless otherwise indicated. MDCT: multidetector-row computed tomography; DVT: deep venous thrombosis; CUS: lower limb compression ultrasonography. ${ }^{\#}$ : death possibly linked to PE.

higher than the $1-2 \%$ risk usually reported in emergency patients [10, 11, 17].

The present results indicate that MDCT still misses small subsegmental clots. However, the clinical relevance of these small PEs is a matter of controversy, with some authors arguing that anticoagulation may be safely withheld in this setting $[19,21]$. Actually, in a large population of in-patients in whom PE had been excluded by CT pulmonary angiography (involving single- and multidetector row CT scans) without further work-up, KRUIP et al. [20] have recently observed a 3 -month risk of VTE of $4 \%$. However, the CI was large (1.5-8\%) and it cannot be excluded that a systematic search for DVT might have reduced the subsequent VTE.

\section{TABLE 3 Clinical outcome in patients considered negative for pulmonary embolism (PE)}

\begin{tabular}{lcc} 
Events & \multicolumn{2}{c}{ Accordance with reference standard } \\
\cline { 2 - 3 } & Yes & No \\
\hline Subjects $\mathbf{n}$ & 169 & 115 \\
Total deaths & $13(7.6)$ & $9(7.8)$ \\
Excluded & $23(13.5)$ & $11(9.6)$ \\
Lost to follow-up & $3(1.8)$ & $7(6.1)$ \\
VTE events & & \\
DVT & $1(0.6)$ & $2(1.7)$ \\
PE & $2(1.2)$ & $4(3.5)$ \\
Death possibly linked to PE & $2(1.2)$ & $1(0.9)$ \\
Total VTE events & $5(3.8)$ & $7(7.9)$ \\
\hline
\end{tabular}

Data presented as n (\%), unless otherwise stated. VTE: venous thromboembolic events; DVT: deep venous thrombosis.
The present study does not make it possible to conclude this debate, since nonadherence with the reference standard doubled the 3-month risk of VTE $(7.9 \%)$, but this difference did not reach statistical significance due to the small number of events. Nevertheless, since small emboli are less likely to cause symptoms in otherwise healthy patients, they are more likely to be fatal in in-patients with multiple risk factors or poor cardiopulmonary reserve $[22,23]$. Therefore, until it is proven that anticoagulation therapy can be safely withheld in patients with small PE, the current authors believe it is prudent to continue to systematically test in-patients with suspected PE for DVT when a negative MDCT provides no alternative diagnosis for patients' signs or symptoms, and to leave to the discretion of the attending physician the decision whether to withhold anticoagulation therapy in selected patients [24]. As a result, in order to improve the management of in-patients with suspected PE, and in accordance with the recent PIOPED recommendations [25], the current authors have replaced in their reference standard the MDCT pulmonary angiography by a combined MDCT pulmonary angiography with $\mathrm{CT}$ indirect venography as the frontline diagnostic test. Since then, $97 \%$ of patients with an MDCT negative for PE are adequately tested for DVT (data not shown). However, irradiation of the pelvis and gonads is an important consideration when performing indirect CT venography, especially in young patients. RADEMAKER et al. [26] have measured patient gonadal doses in the order of $2.1-10.7 \mathrm{mSv}$ in this setting, with variations between individuals and sexes. This increase in gonadal dose is well bellow the threshold for deterministic radiation effects provided in the International Commission on Radiological Protection Publication 60 guidelines [27], and will further decrease with the wider use of a higher number of detector rows and application of dose-modulation programs [28], but is nevertheless significant and needs to be balanced with the better management of suspected PE induced by the use of CT venography in the diagnostic work-up. 
In order to minimise the radiation burden, patients with suspected PE should ideally first undergo CUS of the legs [29]. In the present study, among the 30 patients undergoing CUS before MDCT or lung scan, DVT could be established in eight patients without any radiation exposure. However, this strategy is hampered by the fact that the diagnosis of PE or an alternative diagnosis is delayed in patients with normal CUS, especially in hospitals, such as that of the present authors, where the availability of MDCT is better than that of CUS.

The research undertaken in the present study has several limitations. The first limitation lies in its design. The current study included a large cohort of patients in a real-life setting, avoiding subject selection and response shift bias, but it is limited by the biases inherent in collecting data retrospectively. However, the present authors believe this bias was minimised since their patient selection was nearly exhaustive, no patient was excluded due to missing data, and the number of patients lost to follow-up was small. Secondly, the rate at which physicians appropriately managed patients may have been overestimated, since the guidelines used did not define which patient should undergo additional investigation (perfusion lung scan or a pulmonary digital-subtraction angiography) after a negative firstline diagnostic round. A deliberate choice was made to leave additional investigations to the discretion of the attending physician due to the small number $(<2 \%)$ of patients concerned $[3,19]$, in order to focus on more frequent causes of inappropriate diagnostic management. Thirdly, the potential for overdiagnosis of PE was not evaluated in the present study. MDCTs considered positive for PE were not systematically reviewed by an independent reader and patients did not undergo confirmatory pulmonary angiography. Therefore, although the specificity of MDCT for the diagnosis of PE is considered to be high, the present study design, like most outcome studies, did not allow the assessment of the false-positive rate of pulmonary MDCT.

In summary, it was concluded that a frontline diagnostic workup based on pulmonary multidetector-row computed tomography associated with a test for deep vein thrombosis is effective in ruling out pulmonary embolism in hospitalised patients. It is more sensitive than pulmonary multidetector-row computed tomography alone but this did not translate into a significantly lower incidence of a venous thromboembolic event at follow-up, possibly due to the too-small number of events.

\section{ACKNOWLEDGEMENTS}

The authors are grateful to G. Meyer (Georges Pompidou European Hospital) for his expert advice and helpful reviews of earlier versions of the manuscript, and wish to thank J.C. Masson, D. Patte and E. Merier-Pinatel (CHI Poissy-St Germain, St Germain-en-Laye, France) for their help in data collection.

\section{REFERENCES}

1 Girard P, Decousus M, Laporte S, et al. Diagnosis of pulmonary embolism in patients with proximal deep vein thrombosis. Am J Respir Crit Care Med 2001; 164: 1033-1037.

2 Kruip M, Leclercq M, Van Der Heul C, Prins M, Buller H. Diagnostic strategies for excluding pulmonary embolism in clinical outcome studies. Ann Intern Med 2003; 138: 941-951.
3 Roy P, Meyer G, Vielle B, et al. Appropriateness of diagnostic management and outcomes of suspected pulmonary embolism. Ann Intern Med 2006; 144: 157-164.

4 Oger E. Incidence of venous thromboembolism: a community-based study in western France. Thromb Haemost 2000; 83: 657-660.

5 Brotman D, Segal J, Jani J, Petty B, Kickler T. Limitations of D-dimer testing in unselected inpatients with suspected venous thromboembolism. Am J Med 2003; 114: 276-282.

6 Schrecengost J, LeGallo R, Boyd J, et al. Comparison of diagnostic accuracies in outpatients and hospitalized patients of D-dimer testing for the evaluation of suspected pulmonary embolism. Clin Chem 2003; 49: 1483-1490.

7 Miron M, Perrier A, Bounameaux H, et al. Contribution of noninvasive evaluation to the diagnosis of pulmonary embolism in hospitalized patients. Eur Respir J 1999; 13: 1365-1370.

8 Kearon C. Diagnosis of pulmonary embolism. CMAJ 2003; 168: 183-194.

9 Schoepf U, Holzknecht N, Helmberger T, et al. Subsegmental pulmonary emboli: improved detection with thin collimation multi-detector row spiral CT. Radiology 2002; 222: 483-490.

10 Writing Group for the Christopher Study Investigators. Effectiveness of managing suspected pulmonary embolism using an algorithm combining clinical probability, D-dimer testing, and computed tomography. JAMA 2006; 295: 172-179.

11 Perrier A, Roy P, Sanchez O, et al. Multidetector-row computed tomography in suspected pulmonary embolism. $N$ Engl J Med 2005; 352: 1760-1768.

12 Girard P, Musset D, Parent F, Maitre S, Philippoteau C, Simonneau G. High prevalence of detectable deep vein thrombosis in patients with acute pulmonary embolism. Chest 1999; 116: 903-908.

13 Le Gal G, Righini M, Sanchez O, et al. A positive compression ultrasonography of the lower limb is highly predictive of pulmonary embolism in suspected patients. Thromb Haemost 2006; 95: 963-966.

14 Fedullo P, Tapson V. The evaluation of suspected pulmonary embolism. N Engl J Med 2003; 349: 1247-1256.

15 The PIOPED investigators. Value of the ventilation/ perfusion scan in acute pulmonary embolism. JAMA 1990; 263: 2753-2759.

16 Ghaye B, Nchimi A, Noukoua C, Dodelinger R. Does multi-detector row $\mathrm{CT}$ pulmonary angiography reduce the incremental value of indirect CT venography compared with single-detector row CT pulmonary angiography? Radiology 2006; 240: 256-262.

17 Stein P, Fowler S, Goodman L, et al. Multidetector computed tomography for acute pulmonary embolism. N Engl J Med 2006; 354: 2317-2327.

18 Bourriot K, Couffinhal T, Bernard V, Montaudon M, Bonnet J, Laurent F. Clinical outcome after a negative spiral CT pulmonary angiographic finding in an inpatient population from cardiology and pneumology wards. Chest 2003; 123: 359-365.

19 Musset D, Parent F, Meyer G, et al. Diagnostic strategy for patients with suspected pulmonary embolism: a prospective multicentre outcome study. Lancet 2002; 360: 1914-1920. 
20 Kruip M, Söhne M, Nijkeuter M, et al. A simple diagnostic strategy in hospitalized patients with clinically suspected pulmonary embolism. Ann Intern Med 2006; 260: 459-466.

21 Egermayer P, Town G. The clinical significance of pulmonary embolism: uncertainties and implications for treatment - a debate. J Intern Med 1997; 241: 5-10.

22 Hull R, Raskob G, Pineo G, Brant R. The low probability lung scan: a need for change in the nomenclature. Arch Intern Med 1995; 155: 1845-1851.

23 Goodman L, Lipchik R. Diagnosis of acute pulmonary embolism: time for a new approach. Radiology 1995; 199: 25-27.

24 Goodman L. Small pulmonary embolism: what do we know? Radiology 2005; 234: 654-658.

25 Stein P, Woddart P, Weg J, et al. Diagnostic pathways in acute pulmonary embolism: recommendations of the PIOPED II investigators. Am J Med 2006; 119: 1048-1055.
26 Rademaker J, Griesshaber V, Hidajat N, Oestmann J, Felix R. Combined CT pulmonary angiography and venography for diagnosis of pulmonary embolism and deep vein thrombosis: radiation dose. J Thorac Imaging 2001; 16: 297-299.

27 International Commission on Radiological Protection. 1990 Recommendations of the International Commission on Radiological Protection. ICRP Publication no. 60. Ann ICRP 1991; 21: 67-77.

28 Coche E, Vynckier S, Octave-Prignot M. Pulmonary embolism: radiation dose with multi-detector row CT and digital angiography for diagnosis. Radiology 2006; 240: 690-697.

29 Perrier A, Roy P, Aujesky D, et al. Diagnosing pulmonary embolism in outpatients with clinical assessment, D-dimer measurement, venous ultrasound, and helical computed tomography: a multicenter management study. Am J Med 2004; 116: 291-299. 